\title{
CERTAIN COHOMOLOGY RINGS OF FINITE AND FORMAL GROUP SCHEMES
}

\author{
BY \\ GUSTAVE EFROYMSON
}

Introduction and summary. In [2, I], Demazure defines the cohomology of groups in arbitrary categories. He starts with a category $\mathscr{C}$ and immediately goes over to the "category" $\mathscr{C}$ of contravariant functors $\mathscr{C} \leadsto$ Sets. For a group $G$ in $\mathscr{\mathscr { C }}$ and a $G$-module $F$ in $\mathscr{C}$ (for the definition of these concepts see $\S 1$ ) Demazure can define $H^{*}(G, F)$, the graded cohomology module. In $\S 1$ we repeat this definition and also give the obvious generalization to the case where $F$ is a ring in $\hat{\mathscr{C}}$, then $H^{*}(G, F)$ becomes a graded ring.

Then in $\S 2$, we consider the case of $\mathscr{C}$, the category of finite affine schemes over a commutative ring $k$. When $\mathcal{O}$ is the ring in $\mathscr{\mathscr { C }}$ defined by $\mathcal{O}(\operatorname{Spec} R)=R, G$ is a group scheme, and $G$ acts trivially on $\mathcal{O}$; then $H^{\circ}(G, \mathcal{O})$ is canonically isomorphic to the Hochshild cohomology $H^{*}(A, k)$ for $A$ the affine ring of the Cartier dual of $G$. In fact Theorem 2.1 even connects complexes used to define $H^{*}(G, \mathcal{O})$ and $H^{*}(A, k)$.

In Proposition 2.4 a familiar computation is carried out which enables us to compute $H^{*}(A, k)$ in certain cases. The theorem of Dieudonné-Cartier [2, VIIb] shows that the above mentioned special case is sufficient to compute $H^{*}(G, \mathcal{O})$ when $k$ is a perfect field.

Finally in $\S 3$, the case of formal Lie groups is considered. If $G$ is a divisible formal Lie group over a perfect field $k$ of characteristic $p>2$ with connected "dual", $\mathcal{O}$ the ring element: $\mathcal{O}(\operatorname{Spf} A)=A$, and $G$ acts trivially on $\mathcal{O}$, then

$$
\operatorname{Sym}\left(H_{\text {sym }}^{2}(G, \mathcal{O})\right) \rightarrow H^{\cdot}(G, \mathcal{O})
$$

is an isomorphism of graded $k$-algebras where Sym is the symmetric algebra and $H_{\text {sym }}^{2}$ is defined in $\S 1$. This result was obtained in the author's thesis [3] by different methods. The main motivation for the present research was to find a more natural setting for this result. Remarks by B. Mazur at the occasion of the presentation of the thesis were partly responsible for this search. Also the author wishes to thank J. Tate for his encouragement and helpful advice.

\section{Definition of cohomology of groups.}

Definition 1.1. In our definitions we follow [2, I]. Let $\mathscr{C}$ be a category. Let $\hat{\mathscr{C}}$ be the "category" of contravariant functors $\mathscr{C} \rightarrow$ Sets. Morphisms in $\mathscr{C}$ are just natural transformations of functors. $\hat{\mathscr{C}}$ has a final object $\boldsymbol{e}: \boldsymbol{e}(S)=\{$ one point $\}$ for

Received by the editors September 25, 1968 and, in revised form, April 28, 1969. 
all $S$ in $\mathscr{C}$. To each $C$ in $\mathscr{C}$ we associate $C$ in $\mathscr{C}$ defined by $C(S)=\operatorname{Hom}(S, C)$ for all $S$ in $\mathscr{C}$. It is fairly easily seen that $C \mapsto C$ is a fully faithful functor $\mathscr{C} \leadsto \mathscr{\mathscr { C }}$. Let $X$ be represented by $X \in \mathscr{C}$ so $X(S)=\operatorname{Hom}(S, X)$. Let $F \in \hat{\mathscr{C}}$. Then the map $\beta: \operatorname{Hom}(\boldsymbol{X}, \boldsymbol{F}) \rightarrow \boldsymbol{F}(X)$ is a bijection where for $f \in \operatorname{Hom}(\boldsymbol{X}, \boldsymbol{F}), \beta(f)=\boldsymbol{F}\left(f_{X}\right)\left(1_{X}\right)$ $\left(1_{X}\right.$ is the identity map $\left.X \rightarrow X\right)$.

Definition 1.2. If $\boldsymbol{F}_{1}$ and $\boldsymbol{F}_{2}$ are objects of $\hat{\mathscr{C}}$, then $\boldsymbol{F}_{1} \times \boldsymbol{F}_{2}$ in $\hat{\mathscr{C}}$ is defined by $\left(F_{1} \times F_{2}\right)(S)=F_{1}(S) \times F_{2}(S)$. If $C$ and $D$ are objects of $\mathscr{C}$ and $C \times D$ is representable, we denote the object which represents it by $C \times D$.

Definition 1.3. $G$ in $\mathscr{C}$ is a group object of $\mathscr{C}$ if $G(S)$ is a group for all $S \in \mathscr{C}$; and if for all $f \in \operatorname{Hom}\left(S_{1}, S_{2}\right), \boldsymbol{G}(f): \boldsymbol{G}\left(S_{2}\right) \rightarrow \boldsymbol{G}\left(S_{1}\right)$ is a group homomorphism. $\boldsymbol{G}$ is an abelian group in $\hat{\mathscr{C}}$ if $\boldsymbol{G}(S)$ is abelian for all $S \in \mathscr{C}$. We say $G$ is a group in $\mathscr{C}$ if $\boldsymbol{G}$ is a group in $\hat{\mathscr{C}}, \boldsymbol{G}(S)=\operatorname{Hom}(S, G)$.

Definition 1.4. Let $\boldsymbol{F}$ be an abelian group in $\mathscr{C}, \boldsymbol{G}$ a group in $\hat{\mathscr{C}}$. Then a $\mathscr{C}$ morphism $\mu: \boldsymbol{G} \times \boldsymbol{F} \rightarrow \boldsymbol{F}$ makes $\boldsymbol{F}$ into a $\boldsymbol{G}$-module if, for all $S \in \mathscr{C}, \mu_{S}: \boldsymbol{G}(S) \times \boldsymbol{F}(S)$ $\rightarrow \boldsymbol{F}(S)$ makes $\boldsymbol{F}(S)$ into a $\boldsymbol{G}(S)$-module functorially in $S$.

Definition 1.5. Group Cohomology in $\hat{\mathscr{C}}$. Let $\boldsymbol{G}$ be a group in $\hat{\mathscr{C}}, \boldsymbol{F}$ a $\boldsymbol{G}$-module in $\hat{\mathscr{C}}$. Then $H^{*}(\boldsymbol{G}, \boldsymbol{F})$ is the cohomology of the complex $C^{*}(\boldsymbol{G}, \boldsymbol{F})$ where $C^{n}(\boldsymbol{G}, \boldsymbol{F})$ $=\operatorname{Hom}\left(\boldsymbol{G}^{\times n}, \boldsymbol{F}\right)$ for $n=0,1,2, \ldots, \boldsymbol{G}^{0}=\boldsymbol{e}$, and the boundary map $\delta: C^{n}(\boldsymbol{G}, \boldsymbol{F}) \rightarrow$ $C^{n+1}(\boldsymbol{G}, \boldsymbol{F})$ is defined as follows: If $f_{S}: \boldsymbol{G}^{\times n}(S) \rightarrow \boldsymbol{F}(S)$ defines an element of $C^{n}(\boldsymbol{G}, \boldsymbol{F})$, let $(\delta f)_{S}: \boldsymbol{G}^{\times n+1}(S) \rightarrow \boldsymbol{F}(S)$ be defined by

$$
\begin{aligned}
(\delta f)_{S}\left(g_{1}, \ldots, g_{n+1}\right)= & g_{1} \cdot f_{S}\left(g_{2}, \ldots, g_{n+1}\right) \\
& +\sum_{j=1}^{n}(-1)^{j} f_{S}\left(g_{1}, g_{2}, \ldots, g_{j} g_{j+1}, \ldots, g_{n+1}\right) \\
& +(-1)^{n+1} f_{S}\left(g_{1}, \ldots, g_{n}\right)
\end{aligned}
$$

for $g_{i} \in \boldsymbol{G}(S)$. Addition is in $\boldsymbol{F}(S)$ and $g_{1} \cdot f_{S}\left(g_{2}, \ldots, g_{n+1}\right)$ uses the $\boldsymbol{G}(S)$-module structure on $F(S)$.

Definition 1.6. If $\left(\boldsymbol{G}_{1}, \boldsymbol{F}_{1}\right)$ and $\left(\boldsymbol{G}_{2}, \boldsymbol{F}_{2}\right)$ are pairs consisting of a group $\boldsymbol{G}_{\boldsymbol{i}}$ in $\hat{\mathscr{C}}$ and a $\boldsymbol{G}_{i}$-module $\boldsymbol{F}_{\boldsymbol{i}}$ in $\mathscr{C}$, then $(u, v):\left(\boldsymbol{G}_{1}, \boldsymbol{F}_{1}\right) \rightarrow\left(\boldsymbol{G}_{2}, \boldsymbol{F}_{2}\right)$ is a map of pairs if $u: G_{2} \rightarrow G_{1}$ and $v: F_{1} \rightarrow F_{2}$ are maps such that for all $S \in \mathscr{C}, g \in G_{2}(S), \alpha \in F_{1}(S)$, we have $g \cdot v(\alpha)=v(u(g) \cdot \alpha)$.

Proposition 1.1. A map $(u, v)$ of pairs $\left(\boldsymbol{G}_{1}, \boldsymbol{F}_{1}\right) \rightarrow\left(\boldsymbol{G}_{2}, \boldsymbol{F}_{2}\right)$ as above induces a map $H^{\cdot}\left(\boldsymbol{G}_{1}, \boldsymbol{F}_{1}\right) \rightarrow H^{\cdot}\left(\boldsymbol{G}_{2}, \boldsymbol{F}_{2}\right)$.

Proof. $(u, v)$ induces a map $C^{\cdot}\left(\boldsymbol{G}_{1}, \boldsymbol{F}_{1}\right) \rightarrow C^{\cdot}\left(\boldsymbol{G}_{2}, \boldsymbol{F}_{2}\right)$ of complexes which induces a map of cohomology groups.

Definition 1.7. $F$ in $\hat{\mathscr{C}}$ is a ring object in $\hat{\mathscr{C}}$ if $\boldsymbol{F}(S)$ is a ring for all $S \in \mathscr{C}$ and if for all $f \in \operatorname{Hom}\left(S_{1}, S_{2}\right), \boldsymbol{F}(h): \boldsymbol{F}\left(S_{2}\right) \rightarrow \boldsymbol{F}\left(S_{1}\right)$ is a ring homomorphism.

Definition 1.8. If $\boldsymbol{F}$ is a ring in $\hat{\mathscr{C}}$ and a $\boldsymbol{G}$ module so that the action of $\boldsymbol{G}(S)$ on $F(S)$ is consistent with multiplication in $F(S)$; then one can define

$$
\cup_{*}: C^{n}(\boldsymbol{G}, \boldsymbol{F}) \otimes C^{m}(\boldsymbol{G}, \boldsymbol{F}) \rightarrow C^{n+m}(\boldsymbol{G}, \boldsymbol{F})
$$


by $f_{S} \otimes f_{S}^{\prime} \rightarrow f_{S} \cup_{*} f_{S}^{\prime}$ where

$$
f_{S} \cup_{*} f_{S}^{\prime}\left(g_{1}, \ldots, g_{n+m}\right)=f_{S}\left(g_{1}, \ldots, g_{n}\right) \cdot\left(g_{1} g_{2}, \ldots, g_{n}\right) f_{S}^{\prime}\left(g_{n+1}, \ldots, g_{n+m}\right)
$$

and where the dot denotes multiplication in the ring $F(S)$.

PROPOSITION 1.2. The map $\cup_{*}$ defined above induces a graded ring structure on $H^{*}(G, F)$ with multiplication denoted by $\cup$ and this graded ring is commutative if $\boldsymbol{F}$ is commutative. Commutative means that if $f \in H^{n}(\boldsymbol{G}, \boldsymbol{F}), f^{\prime} \in H^{m}(\boldsymbol{G}, \boldsymbol{F})$, then $f \cup f^{\prime}=(-1)^{m n} f^{\prime} \cup f$ in $H^{m+n}(\boldsymbol{G}, \boldsymbol{F})$.

Proof. We can use the usual homotopies on $C^{n}(G, F)(S)=\operatorname{Hom}\left(G^{\times n}(S), F(S)\right)$. Thus to show commutativity we have

$$
t_{S}: C^{n}(\boldsymbol{G}, \boldsymbol{F})(S) \otimes C^{m}(\boldsymbol{G}, \boldsymbol{F})(S) \rightarrow C^{n+m-1}(\boldsymbol{G}, \boldsymbol{F})(S)
$$

so that $\left(f \cup_{*} f^{\prime}-f^{\prime} \cup_{*} f\right)\left(g_{1}, \ldots, g_{n+m}\right)=\delta\left(t_{s}\left(f \otimes f^{\prime}\right)\right)\left(g_{1}, \ldots, g_{n+m}\right)$. The other axioms for a ring can be similarly demonstrated.

Proposition 1.3. A map of pairs $(u, v):\left(\boldsymbol{G}_{1}, \boldsymbol{F}_{1}\right) \rightarrow\left(\boldsymbol{G}_{2}, \boldsymbol{F}_{2}\right)$ (Definition 1.6) induces a graded ring homomorphism $H^{*}\left(G_{1}, F_{1}\right) \rightarrow H^{*}\left(G_{2}, F_{2}\right)$.

Proof. This should be clear.

Proposition AND Definition 1.4. Let $\boldsymbol{G}$ be an abelian group in $\hat{\mathscr{C}}$, let $\boldsymbol{F}$ be an abelian group in $\hat{\mathscr{C}}$ and let $\boldsymbol{G}$ act trivially on $\boldsymbol{F}$.

Let $C_{\mathrm{sym}}^{2}(\boldsymbol{G}, \boldsymbol{F})=\left\{f \in \operatorname{Hom}\left(\boldsymbol{G}^{\times 2}, \boldsymbol{F}\right)\right.$ such that for all $S$ in $\mathscr{C}$ and all $g_{1}, g_{2}$ in $\left.\boldsymbol{F}(S) ; f_{\mathrm{S}}\left(g_{1}, g_{2}\right)=f_{S}\left(g_{2}, g_{1}\right)\right\}$. Let $Z_{\mathrm{sym}}^{2}(\boldsymbol{G}, \boldsymbol{F})=\left\{f \in C_{\mathrm{sym}}^{2}(\boldsymbol{G}, \boldsymbol{F}) \mid \delta f=0\right\}$. Then

$$
\delta C^{1}(\boldsymbol{G}, \boldsymbol{F}) \subseteq Z_{\mathrm{sym}}^{2}(\boldsymbol{G}, \boldsymbol{F})
$$

and so we can define $H_{\mathrm{sym}}^{2}(G, F)=Z_{\mathrm{sym}}^{2}(G, F) / \delta C^{1}(G, F)$.

Proof. For $f \in C^{1}(\boldsymbol{G}, \boldsymbol{F})$,

$$
(\delta f)_{S}\left(g_{1}, g_{2}\right)=f_{S}\left(g_{2}\right)-f_{S}\left(g_{1} g_{2}\right)+f_{S}\left(g_{1}\right)=(\delta f)_{S}\left(g_{2}, g_{1}\right)
$$

since $G$ is abelian.

2. Cohomology of finite group schemes and the relation with the cohomology of cocommutative Hopf algebras.

Notation. Let $k$ be a fixed commutative ring with unit element 1 . Let $\mathscr{C}$ be the category of finite affine schemes over $k$ so $X \in \mathscr{C}$ if $X=\operatorname{spec} E$ where $E$ is a finite $k$-algebra.

$$
\mathcal{O}: \mathscr{C} \rightarrow \text { Rings is the functor } \mathcal{O}(\operatorname{Spec} E)=E .
$$

Definition 2.1. A finite group scheme is an element $G$ of $\mathscr{C}$ such that the associated functor $\boldsymbol{G}, \boldsymbol{G}(S)=\operatorname{Hom}(S, G)$ for all $S \in \mathscr{C}$, is a group in $\hat{\mathscr{C}}$. We also require that if $G=\operatorname{Spec} E$, then $E$ must be free as a $k$-module. 
If $G=\operatorname{Spec} E$ is a finite group scheme, then

corresponds to a map

$$
\boldsymbol{G} \times \boldsymbol{G} \stackrel{m_{\boldsymbol{G}}}{\longrightarrow} \boldsymbol{G}
$$

$$
G \times G \stackrel{m_{G}}{\longrightarrow} G
$$

which corresponds in turn to a map $\mu: E \rightarrow E \otimes_{k} E$. The map $\mu$ together with multiplication in $E: E \otimes_{k} E \rightarrow E$ makes $E$ into a Hopf algebra. $E$ is a $k$-algebra via $\sigma^{\prime}: k \rightarrow E$. Also $\varepsilon^{\prime}: E \rightarrow k$ corresponds to the unit element map $\operatorname{Spec} k \rightarrow \operatorname{Spec} E$. Then if $A=\operatorname{Hom}_{k \text {-modules }}(E, k)$ we get induced maps

$$
A \stackrel{\text { mult* }}{\longrightarrow} A \otimes_{k} A \stackrel{\mu^{*}}{\longrightarrow} A,
$$

and $A$ becomes a cocommutative Hopf algebra. We also have maps $\sigma^{\prime *}: A \rightarrow k$, $\varepsilon^{* *}: k \rightarrow A$. If $G$ is a commutative group scheme, Spec $A=G^{\prime}$ is called the Cartier dual of $G$.

Definition 2.2. If $A$ is a $k$-algebra with map $\varepsilon: A \rightarrow k$ making $k$ into an $A$ module, the Hochschild cohomology $H^{\circ}(A, k)$ is defined, for instance in [1, p. 169].

This can be defined or computed from the standard resolution $C .(A, k)$ of $k$ as an $A$-module, where $C_{n}(A, k)=A^{\otimes n+1}$ considered as an $A$-module via the first variable:

$$
a\left(a_{0} \otimes \cdots \otimes a_{n}\right)=a a_{0} \otimes a_{1} \otimes \cdots \otimes a_{n} ; \quad a_{i}, a \in A .
$$

The boundary map $\partial: C_{n}(A, k) \rightarrow C_{n-1}(A, k)$ is defined by

$$
\begin{aligned}
\partial\left(a_{0} \otimes a_{1} \otimes \cdots \otimes a_{n}\right)= & \sum_{j=0}^{n=1}(-1)^{j} a_{0} \otimes \cdots \otimes a_{j} a_{j+1} \otimes \cdots \otimes a_{n} \\
& +(-1)^{n} \varepsilon\left(a_{n}\right) a_{0} \otimes \cdots \otimes a_{n-1} .
\end{aligned}
$$

Then if $\operatorname{Hom}_{A}\left(C_{n}(A, k), k\right)=C^{n}(A, k), C^{*}(A, k)$ has a coboundary $\delta$ defined by $\delta f=f \circ \partial$. The cohomology of $C^{\cdot}(A, k)$ is $H^{\cdot}(A, k)$.

Proposition 2.1. If $A$ is a Hopf algebra with counit $\varepsilon: A \rightarrow k$, then $\Delta: A \rightarrow A \otimes_{k} A$ induces a map $C_{.}(A, k) \rightarrow C_{.}(A, k) \otimes C_{.}(A, k)$ of graded modules which induces $a$ product structure on $H^{\circ}(A, k)$ making it into a graded ring ( $\Delta$ is the coalgebra map, $C .(A, k)$ as in Definition 2.3).

Proof. Well known.

THEOREM 2.1. Let $G=\operatorname{Spec} E, A=\operatorname{Hom}_{k}(E, k)$ as in Definition 2.2, $\varepsilon=\sigma^{*}$, $\sigma=\varepsilon^{\prime *}$. Let $\mathcal{O}$ be the previously defined ring in $\hat{\mathscr{C}}$. Consider $\mathcal{O}$ as a $\boldsymbol{G}$ module with trivial action. Then $C^{\cdot}(G, \mathcal{O})$ and $C^{*}(A, k)$ are canonically isomorphic as complexes. The product structures on the complexes induce isomorphic graded ring structures on the cohomology modules.

Proof. In 1.1 we defined $\beta: C^{n}(G, \mathcal{O})=\operatorname{Hom}\left(G^{\times n}, \mathcal{O}\right) \rightarrow \mathcal{O}\left(G^{\times n}\right)=E^{\otimes n}$. 
The boundary map $\delta: C^{n}(G, \mathcal{O}) \rightarrow C^{n+1}(G, \mathcal{O})$ induces $\beta(\delta): \mathcal{O}\left(G^{\times n}\right) \rightarrow \mathcal{O}\left(G^{\times n+1}\right)$ which is just $\sum_{i=1}^{n+1}(-1)^{i} \mathcal{O}\left(\psi_{i}\right)$.

$$
\begin{aligned}
& \psi_{0}: G^{\times n+1} \rightarrow G^{\times n} \text { is just } \sigma^{\prime} \times 1_{G} \times \cdots \times 1_{G}, \\
& \psi_{i}: G^{\times n+1} \rightarrow G^{\times n} \text { is } 1_{G} \times \cdots \times m_{G} \times \cdots \times 1_{G}, \quad i=1, \ldots, n, \\
& \psi_{n+1}: G^{\times n+1} \rightarrow G^{\times n} \quad \text { is } 1_{G} \times \cdots \times 1_{G} \times \sigma^{\prime},
\end{aligned}
$$

so the map $\sum_{i=0}^{n+1}(-1)^{i} \mathcal{O}\left(\psi_{i}\right)$ becomes:

$$
\begin{aligned}
& \text { jth place } \\
& \sigma^{\prime} \otimes 1_{E} \otimes \cdots \otimes 1_{E}+\sum_{j=1}^{n}(-1)^{j} 1_{E} \otimes \cdots \otimes \mu \otimes \cdots \otimes 1_{E} \\
& +(-1)^{n+1} 1_{E} \otimes \cdots \otimes 1_{E} \otimes \sigma^{\prime} .
\end{aligned}
$$

The other complex

$$
C^{n}(A, k)=\operatorname{Hom}_{A}\left(C_{n}(A, k), k\right)=\operatorname{Hom}_{A}\left(A^{\otimes n+1}, k\right) \stackrel{\theta}{\longrightarrow} \operatorname{Hom}_{k}\left(A^{\otimes n}, k\right),
$$

where for $f \in \operatorname{Hom}_{A}\left(A^{\otimes(n+1)}, k\right), \theta(f) \in \operatorname{Hom}_{k}\left(A^{\otimes n}, k\right)$ and

$$
\theta(f)\left(a_{1} \otimes \cdots \otimes a_{n}\right)=f\left(1 \otimes a_{1} \otimes \cdots \otimes a_{n}\right) .
$$

But $\operatorname{Hom}_{k}\left(A^{\otimes n}, k\right)=E^{\otimes n}$ under the identification of $E$ with $\operatorname{Hom}_{k}(A, k)$ using the facts that $A=\operatorname{Hom}_{k}(E, k)$ and $E$ is finite and free over $k$.

That is, for $f_{1} \otimes \cdots \otimes f_{n} \in E^{\otimes n}, a_{1} \otimes \cdots \otimes a_{n} \in A^{\otimes n}$,

$$
\left(f_{1} \otimes \cdots \otimes f_{n}\right)\left(a_{1} \otimes \cdots \otimes a_{n}\right)=f_{1}\left(a_{1}\right) f_{2}\left(a_{2}\right) \cdots f_{n}\left(a_{n}\right) .
$$

By definition $\delta f=f \circ \partial$ for $f \in C^{n}(A, k)$, so

$$
\begin{aligned}
& \delta\left(f_{1} \otimes \cdots \otimes f_{n}\right)\left(a_{1} \otimes \cdots \otimes a_{n+1}\right) \\
& =\left(f_{1} \otimes \cdots \otimes f_{n}\right) \circ \partial\left(a_{1} \otimes \cdots \otimes a_{n+1}\right) \\
& =\left(f_{1} \otimes \cdots \otimes f_{n}\right)\left[\varepsilon\left(a_{1}\right) a_{2} \otimes \cdots \otimes a_{n+1}+\sum_{j=1}^{n}(-1)^{j} a_{1} \otimes \cdots \otimes a_{j} a_{j+1} \otimes \cdots\right. \\
& \left.\otimes a_{n+1}+(-1)^{n+1} \varepsilon\left(a_{n+1}\right) a_{1} \otimes \cdots \otimes a_{n}\right] \text {. }
\end{aligned}
$$

This implies

$$
\begin{gathered}
\delta\left(f_{1} \otimes \cdots \otimes f_{n}\right)=\left(f_{1} \otimes \cdots \otimes f_{n}\right)\left[\varepsilon \otimes 1_{A} \otimes \cdots \otimes 1_{A}+\sum_{j=1}^{n}(-1)^{j} 1_{A} \otimes \cdots\right. \\
\left.\otimes \mu^{*} \otimes \cdots \otimes 1_{A}+(-1)^{n+1} 1_{A} \otimes \cdots \otimes 1_{A} \otimes \varepsilon\right] \\
=\left[\varepsilon^{*} \otimes 1_{E} \otimes \cdots \otimes 1_{E}+\sum_{j=1}^{n}(-1)^{j} 1_{E} \otimes \cdots\right. \\
\left.\otimes \mu \otimes \cdots \otimes 1_{E}+(-1)^{n+1} 1_{E} \otimes \cdots \otimes 1_{E} \otimes \varepsilon^{*}\right]\left(f_{1} \otimes \cdots \otimes f_{n}\right) .
\end{gathered}
$$

$1_{E}=$ identity map $E \rightarrow E$ and $\varepsilon^{*}=\sigma^{\prime}$. So the two complexes are the same. 
To see that the product structures induced on $H^{*}(A, k)$ and $H^{\circ}(G, \mathcal{O})$ correspond under the identification of these two complexes just defined, we use the Bar resolution $\beta$. $(A, k)$ for the $A$-module $k$ and the next two propositions.

Definition 2.4. Let $I=$ kernel of $\varepsilon: A \rightarrow k$. Then $A=k \oplus I$ as a $k$-module.

Proposition 2.2. If $\Delta: A \rightarrow A \otimes_{k} A$ is the coproduct map, then for $a \in I$, $\Delta a-1 \otimes a-a \otimes 1$ is in $I \otimes I$.

Proof. Since $\varepsilon: A \rightarrow k$ corresponds to $\varepsilon: \operatorname{Spec} k \rightarrow \operatorname{Spec} A=G^{\prime}$ and $\varepsilon$ is the unit element for $G^{\prime}$, we have

$$
G^{\prime} \stackrel{\varepsilon \times 1}{\longrightarrow} G^{\prime} \times G^{\prime} \stackrel{\stackrel{m_{G^{\prime}}}{\longrightarrow}}{\longrightarrow} G^{\prime} \text { commutes. }
$$

Then $[(\varepsilon \otimes 1) \circ \Delta](a)=(\varepsilon \otimes 1)(1 \otimes a)$ for all $a \in A$ which implies $\Delta a-1 \otimes a$ is in $I \otimes A=$ kernel of $\varepsilon \otimes 1$. Similarly $\Delta a-a \otimes 1$ is in $A \otimes I$. So if $a$ is in $I$, $(\Delta a-1 \otimes a-a \otimes 1)$ is in (kernel of $\varepsilon \otimes 1) \cap($ kernel of $1 \otimes \varepsilon)=I \otimes I$.

Proposition 2.3. Keep the notation as above and let $\beta_{n}(A, k)=A \otimes(A / k)^{\otimes n}$ $\cong A \otimes I^{\otimes n}$ considered as an $A$-module via the first variable $A$.

The boundary map $\partial: \beta_{n}(A, k) \rightarrow \beta_{n-1}(A, k)$ is the same as for the standard resolution.

Then $\Delta: A \rightarrow A \otimes_{k} A$ induces a map also called $\Delta$,

$$
\Delta: \beta_{n}(A, k) \rightarrow \sum_{n_{1}+n_{2}=n} \beta_{n_{1}}(A, k) \otimes_{k} \beta_{n_{2}}(A, k)
$$

so that if $\beta_{+}=\sum_{n>0} \beta_{n}$ then

$$
\Delta\left[a_{1}|\cdots| a_{n}\right]=\sum_{i=0}^{n}\left[a_{1}|\cdots| a_{i}\right] \otimes\left[a_{i+1}|\cdots| a_{n}\right] \bmod \beta_{+} \otimes I \beta
$$

where $\left[a_{1}|\cdots| a_{n}\right]=a_{1} \otimes \cdots \otimes a_{n} \in I^{\otimes n}$.

Proof. This is a simple computation using explicit formulas in [1, Chapter XI]. We will do a similar computation in the next proposition. The map $\Delta$ is defined inductively on $\beta_{n}$ by $\Delta=(s \otimes 1+\sigma \varepsilon \otimes 1) \circ \Delta \circ \partial$ where $s$ is a homotopy for $\beta_{.}(A, k)$, $s\left(a\left[a_{1}|\cdots| a_{n}\right]\right)=\left[a a_{1}|\cdots| a_{n}\right]$.

Corollary. Let $\beta^{n}(A, k)=\operatorname{Hom}_{A}\left(\beta_{n}(A, k), k\right)$. Define the boundary map $\delta$ by $\delta f=f \circ \partial$. There is a product map $\cup^{\prime}$ on $\beta^{*}(A, k)$ induced by $\Delta$. Then $f \cup^{\prime} f^{\prime}=$ $\left(f \otimes f^{\prime}\right) \circ \Delta$ for $f \in \beta^{n_{1}}(A, k), f^{\prime} \in \beta^{n_{2}}(A, k)$. Thus $f \cup^{\prime} f^{\prime}$ is in $\beta^{n_{1}+n_{2}}(A, k)$ and $\left(f \cup^{\prime} f^{\prime}\right)\left(\left[a_{1}|\cdots| a_{n_{1}+n_{2}}\right]\right)=f\left(\left[a_{1}|\cdots| a_{n_{1}}\right]\right) f^{\prime}\left(\left[a_{n_{1}+1}|\cdots| a_{n_{1}+n_{2}}\right]\right)$.

Proof. This is clear and the proof of Theorem 2.1 is complete since this product is the same as the product structure on the subcomplex of $C^{*}(G, \mathcal{O})$ corresponding to the Bar resolution, $\beta^{*}(A, k)=\operatorname{Hom}_{k}(\beta .(A, k), k)$. 
We now consider $A$ of the following form:

$$
A \cong k[x] / x f(x) \quad \text { where } f(x)=\sum_{i=0}^{n-1} a_{i} x^{i}, a_{n-1}=1 .
$$

Let $\Delta: A \rightarrow A \otimes A$ be the commutative comultiplication.

Let $\varepsilon: A \rightarrow k$ be the counit, $(\varepsilon(x)=0)$.

Let $\sigma: k \rightarrow A$ be the homomorphism making $A$ a $k$-algebra, $(\sigma(1)=1)$.

Let $\Lambda(T)$ be the exterior algebra in one generator $T$ of degree 1 .

Let $\Gamma(S)$ be the divided powers on one generator $S$ of degree 2 so

$$
\begin{aligned}
\Gamma(S) & =k\left[S_{0}, S_{1}, S_{2}, \ldots\right], \quad \begin{array}{r}
S_{0}=1, S_{1}=S, \\
S_{u} \cdot S_{v}
\end{array}=(u, v) S_{u+v} \quad \text { and } \quad(u, v)=(u+v !) / u ! v ! .
\end{aligned}
$$

Take $X^{\cdot}(A, k)$ to be the algebra $A \otimes \Lambda(T) \otimes \Gamma(S)$. Then one sees

$$
X_{2 r}(A, k)=A \otimes S_{r}, \quad X_{2 r+1}(A, k)=A \otimes S_{r} T .
$$

Now define an $A$-algebra map $\partial: X_{m} \rightarrow X_{m-1}$ by

$$
\partial\left(S_{r} T\right)=x S_{r}, \quad \partial\left(S_{r}\right)=f(x) T S_{r-1} .
$$

Define a $k$-linear map $s: X_{m} \rightarrow X_{m+1}$ by setting

$$
s\left(x^{j} S_{r}\right)=x^{j-1} T S_{r} \text { for } 0<j \leqq n-1, \quad s\left(S_{r}\right)=0,
$$

and

$$
s\left(x^{n-1} S_{r-1} T\right)=S_{r}, \quad s\left(x^{j} S_{r-1} T\right)=0 \quad \text { if } j<n-1 .
$$

Then one checks that $\partial$ is a boundary map and $s$ is a homotopy. Now let $Y^{\cdot}(A, k)$ $=\operatorname{Hom}_{A}(X .(A, k), k)$ with boundary $\delta$ :

$$
\delta f=f \circ \partial .
$$

Proposition 2.4. Let $A$ be an algebra of the form $k[x] /(x f(x))$ as above. Let $Y^{\cdot}(A, k)$ be as above.

Then $Y^{\cdot}(A, k) \cong k[V, U] /\left(V^{2}-a_{1} U\right)$ as a graded algebra where $\operatorname{deg} U=2$, $\operatorname{deg} V=1$ and $\delta U=0, \delta V=a_{0} U$. Moreover $H^{*}(A, k)$ is the cohomology of $Y^{\cdot}(A, k)$.

Proof. By the preliminary remarks we know $H^{*}(A, k)$ is the cohomology of $Y^{*}(A, k)$. To compute multiplication in $Y^{*}(A, k)$ we must compute comultiplication in $X .(A, k)$. Let $I=\operatorname{ker} \varepsilon$ as in Proposition 2.4 and $X_{+}=\sum_{n>0} X_{n}(A, k)$. Then

$\left(\mathrm{a}_{\mathrm{r}}\right) \quad \Delta S_{r}=\sum_{u+v=r} S_{u} \otimes S_{v}+\sum_{u+v=r-1} \frac{f(x)-a_{0}}{x} T S_{u} \otimes T S_{v} \bmod \left(X_{+} \otimes I X\right)_{2 r}$,

(br) $\quad \Delta S_{r} T=\sum_{u+v=r} T S_{u} \otimes S_{v}+S_{u} \otimes T S_{v} \bmod \left(X_{+} \otimes I X\right)_{2 r+1}$ 
To demonstrate $\left(a_{r}\right)$ and $\left(b_{r}\right)$ we proceed by induction and use the facts that $s \otimes 1+\sigma \varepsilon \otimes s$ is a homotopy for $X . \otimes X$. ([1], p. 214) and

$$
(s \otimes 1+\sigma \varepsilon \otimes s)\left(X_{+} \otimes I X .\right) \subseteq X_{+} \otimes I X .
$$

since $\sigma \varepsilon=0$ on $X_{+}$.

We use $\Delta=(s \otimes 1+\sigma \varepsilon \otimes 1) \circ \Delta \circ \partial$ to define $\Delta$ by induction on degree ([1] p. 215, Proposition 5.2).

$$
\left(a_{r}\right) \Rightarrow\left(b_{r}\right) \text { : }
$$

$$
\Delta\left(S_{r} T\right)=(s \otimes 1+\sigma \varepsilon \otimes 1) \circ \Delta \circ \partial S_{r} T=(s \otimes 1+\sigma \varepsilon \otimes 1) \circ \Delta\left(x S_{r}\right) ;
$$

but

$$
\Delta\left(x S_{r}\right)=\Delta x \cdot \Delta S_{r}=(x \otimes 1+1 \otimes x) \Delta S_{r} \bmod X_{+} \otimes I X .+I \otimes I X .
$$

and

$$
(s \otimes 1+\sigma \varepsilon \otimes 1)(I \otimes I X .) \subseteq X_{+} \otimes I X .
$$

so

$$
\begin{aligned}
\Delta\left(S_{r} T\right)=(s \otimes 1+\sigma \varepsilon \otimes s) & {\left[\sum_{u+v=r} x S_{u} \otimes S_{v}+S_{u} \otimes x S_{v}\right.} \\
& \left.+\sum_{u+v=r-1}\left(f(x)-a_{0}\right) T S_{u} \otimes T S_{v}\right] \bmod X_{+} \otimes I X . \\
= & \sum_{u+v=r} T S_{u} \otimes S_{v}+1 \otimes T S_{r}+\sum_{u+v=r-1} S_{u+1} \otimes T S_{v} \bmod X_{+} \otimes I X .
\end{aligned}
$$

$\left(b_{r}\right) \Rightarrow\left(a_{r}\right):$

$$
\begin{aligned}
\Delta S_{r+1} & =(s \otimes 1+\sigma \varepsilon \otimes s) \circ \Delta \circ \partial S_{r+1}=(s \otimes 1+\sigma \varepsilon \otimes s) \\
\Delta\left(f(x) T S_{r}\right) & =(s \otimes 1+\sigma \varepsilon \otimes s)\left(\Delta f(x) \cdot \Delta T S_{r}\right), \\
\Delta f(x) & =\sum_{i=0}^{n-1} a_{i}\left(1 \otimes x^{i}+x^{i} \otimes 1\right) \bmod I \otimes I
\end{aligned}
$$

so

$$
\begin{aligned}
\Delta S_{r+1}=(s \otimes 1+\sigma \varepsilon \otimes 1) & {\left[\sum_{u+v=r} x^{n-1} T S_{u} \otimes S_{v}\right.} \\
& \left.+\sum_{u+v=r} f(x) S_{u} \otimes T S_{v}+1 \otimes x^{n-1} T S_{r}\right] \bmod X_{+} \otimes I X . \\
= & \sum_{u+v=r} S_{u+1} \otimes S_{v}
\end{aligned}
$$

$+1 \otimes S_{r+1} \bmod X_{+} \otimes I X$.

Now $\cup^{\prime}: \operatorname{Hom}_{A}(X, k) \otimes \operatorname{Hom}_{A}(X, k) \rightarrow \operatorname{Hom}_{A}(X, k)$ is just $f \cup^{\prime} g=(f \otimes g) \circ \Delta$. Since $f \otimes g$ vanishes on $I X . \otimes X_{.}+X_{.} \otimes I X$, we have not ignored any significant terms.

To finish the computation, noting $\operatorname{Hom}_{A}\left(X_{i}, k\right) \cong k$ all $i$, we can let $V$ be a basis 
for $Y^{1}(A, k)=\operatorname{Hom}_{A}\left(X_{1}, k\right)$ dual to $T$ and let $U$ be a basis for $Y^{2}(A, k)$ dual to $S$. Then $\left(V \cup^{\prime} V\right)(S)=(V \otimes V)(\Delta S)=(V \otimes V)\left(S \otimes 1+1 \otimes S+a_{1} T \otimes T\right)=a_{1}$ so

$$
V \cup^{\prime} V=a_{1} U
$$

and other computations for $U^{\prime}$ are similar.

Moreover $(\delta V)(S)=V(\partial S)=V(f(x) T)=a_{0}$ and so $\delta V=a_{0} U .(\delta U)(S T)=U(\partial S T)$ $=U(x S)=0$ so $\partial U=0$ and $\delta$ can be shown to be a derivation on

$$
k[U, V] /\left(V^{2}-a_{1} U\right)=Y^{\cdot}(A, k) .
$$

REMARK. In addition it can be shown that if $\theta$ is the usual map:

$$
k[U, V] /\left(V^{2}-a_{1} U\right) \rightarrow C^{\cdot}(A, k),
$$

the standard complex, then $\theta(U) \in C_{\mathrm{sym}}^{2}(A, k)$.

Corollary. If $A \cong k[x] / x f_{1}(x) \otimes \cdots \otimes k[x] / x f_{t}(x), f_{i}(x)=\sum_{j=0}^{n_{i}} a_{i j} x^{j}, a_{i n_{i}}=1$ and $A$ is a cocommutative Hopf algebra with counit $\varepsilon: A \rightarrow k$; then $H^{*}(A, k)$ is the cohomology of the complex $Y^{\cdot}(A, k)=k\left[U_{1}, \ldots, U_{t}, V_{1}, \ldots, V_{t}\right], \quad V_{i}^{2}=a_{i 1} U_{i}$, $V_{i} V_{j}=-V_{j} V_{i}$ for $i \neq j$, and $U_{i} V_{j}=V_{j} U_{i}, U_{i} U_{j}=U_{j} U_{i}$ all $i$ and $j, \delta U_{i}=0, \delta V_{i}=a_{0} U_{i}$ $U_{i}$ of degree 2, $V_{i}$ of degree 1. Moreover for the map $\theta: Y^{\cdot}(A, k) \rightarrow C^{*}(A, k)$, $\theta\left(U_{i}\right) \in C_{\text {sym }}^{2}(A, k)$.

Proof. Let $A_{i}=k[x] / x f_{i}(x)$. If $X_{.}^{(i)}\left(A_{i}, k\right)=A_{i} \otimes \Lambda\left(T_{i}\right) \otimes \Gamma\left(S_{i}\right), i=1, \ldots, t$, then $X_{\cdot}^{(1)} \otimes \cdots \otimes X_{\cdot}^{(t)}$ is an $A$-resolution of $k$ and $\operatorname{Hom}_{A}\left(X_{\cdot}^{(1)} \otimes \cdots \otimes X_{\cdot}^{(t)}, k\right) \cong Y^{\cdot}(A, k)$ as above.

Now let $k$ be a perfect field of characteristic $p$. Let $G=\operatorname{Spec} E$ be a finite commutative group scheme over $k$. Then $G \cong G^{0} \times G^{\text {et }}$ where $G^{0}$ is connected and $G^{\text {et }}$ is étale so that if $G=G^{0}, E \cong k\left[x_{1}, \ldots, x_{d}\right] /\left(x_{1}^{p^{r} 1}, \ldots, x_{d}^{p^{\tau} d}\right)$ by the theorem of Cartier-Dieudonné [2, VIIb, p. 152].

THEOREM 2.2. Let $G$ be a finite commutative group scheme over a perfect field $k$ of characteristic $p>0$. Then $H^{*}(G, \mathcal{O})$ is a commutative graded algebra with multiplication given by the cup product.

(a) If $p \neq 2$, this graded ring is freely generated by $H^{1}(G, \mathcal{O})$ and $H_{\mathrm{sym}}^{2}(G, \mathcal{O})$. Freely generated means that the only relations are $f \cup g=(-1)^{m n} g \cup f$ for $f$ in $H^{n}(G, \mathcal{O})$ and $g$ in $H^{m}(G, \mathcal{O})$.

(b) If $p=2$, there are subspaces $V_{1}, V_{2}$ of $H^{1}(G, \mathcal{O})$ and $V_{3}$ of $H_{\mathrm{sym}}^{2}(G, \mathcal{O})$ such that $H^{\cdot}(G, \mathcal{O})$ is generated by $V_{1}, V_{2}, V_{3}$ with the additional relations: $f \cup f=0$ for all $f$ in $V_{1}$.

Proof. Let $G^{\prime}=$ Spec $A$ be the Cartier dual of $G$. Then by Theorem 2.1, $C^{\cdot}(G, \mathcal{O})$ can be identified with $C^{\cdot}(A, k)$. By $[4, \S 3] G^{\prime} \cong G_{\text {et }}^{\prime} \times G_{\text {loc }}^{\prime}$ where $G_{\text {et }}^{\prime}=\operatorname{Spec} A_{\text {et }}$ and $G_{\text {loc }}^{\prime}=\operatorname{Spec} A_{\text {loc }}$.

$A_{\text {et }}$ is an étale $k$-algebra and $A_{\text {loc }}$ is a local $k$-algebra. Moreover both $A_{\text {et }}$ and $A_{\text {loc }}$ have coalgebra structure so that $H^{\cdot}(A, k) \cong H^{*}\left(A_{\text {loc }}, k\right) \otimes H^{*}\left(A_{\text {et }}, k\right)$. But $H^{*}\left(A_{\text {et }}, k\right)$ 
$=0$ by $[2, \mathrm{I}]$ or more directly by the following argument. Let $\bar{k}$ be an algebraic closure of $k$. Let $A=A_{\text {ct }} \otimes_{k} \bar{k}$. Then $H^{n}\left(A_{\text {et }}, k\right) \otimes \bar{k} \cong H^{n}(\bar{A}, \bar{k})$ by the universal coefficient theorem. But $\operatorname{Spec} \bar{A}$ is an étale group scheme. Thus $\bar{A} \cong \bar{k}[H]$ as an algebra for some finite abelian group $H$ of order prime to $p([4, \S 3])$. Now use the fact that $H$ is isomorphic to a product of cyclic groups of order prime to $p$. We are in position to use Proposition 2.4 to see that $H^{n}(\bar{A}, \bar{k})=0$ if $n>0$ and $H^{0}(\bar{A}, \bar{k})$ $=\bar{k}$. This implies $H^{\circ}\left(A_{\text {et }}, k\right)=k$.

Now we can assume $A=A_{\text {loc }}$. The theorem of Dieudonné-Cartier [2, p. 152] tells us that $A \cong k[H]$ as an algebra where $H$ is now a finite group whose order is a power of $p$. Since $H$ is a product of cyclic groups of $p$-power order, we can write $A$ as a tensor product of algebras of the form $k[x] /\left(x^{p^{r}}\right)$. By Proposition 2.4 such an algebra has cohomology equal to the cohomology of the complex $k[V, U] /\left(V^{2}-a_{1} U\right)$, $V$ of degree 1, $U$ of degree 2 and $\delta U=\delta V=0$. We have two subcases: (a) If either $p>2$, or $p=2$ and $r>1$; then $a_{1}=0$. Then the cohomology graded ring is just $k[V, U]$. (b) If $p=2$ and $r=1$, then $a_{1}=1$ and the graded cohomology ring is just $k[V]$.

Take the tensor product of the appropriate complexes. By the Künneth theorem we get $H^{\cdot}(A, k)$ as the cohomology of this complex. Recall that the $U$ 's correspond to elements of $H_{\mathrm{sym}}^{2}(A, k)$.

3. Formal Lie groups. Let $\mathscr{C}$ be the category of formal affine local schemes over $k$, a field. Thus, an object of $\mathscr{C}$ will be $\operatorname{Spec}$ formal $A=\operatorname{Spf} A$ where $A$ is a local ring with maximal ideal $m$ and $A$ is complete with respect to the $m$-adic topology.

Definition 3.1. A group in $\mathscr{C}$ (see Definition 1.3) is called a formal group.

If $G=\operatorname{Spf} A, A=k\left[\left[x_{1}, \ldots, x_{d}\right]\right], G$ a formal group, then $G$ is called a formal Lie group.

Definition 3.2. For any commutative group scheme $G$, the map $m_{G}: G \times G \rightarrow G$ induces a map $[n]: G \rightarrow G$ defined inductively on $G(S)$ by $[n] g=[n-1] g \cdot g$ for all $g \in \boldsymbol{G}(S)$.

Definition 3.3 (TATE [6]). If $k$ is of characteristic $p$ and $G$ a commutative formal Lie group such that kernel $[p]$ (=kernel of the map $[p]: G \rightarrow G)$ is a finite group scheme, then $G$ is called a divisible formal Lie group.

The category of finite abelian group schemes over a field is abelian [5] or [2, VIa]. Thus we are within our rights to consider kernels and exact sequences in this category.

Definition 3.4 (TATE [6]). Let $h$ be a positive integer. A sequence $\left(G_{r}, i_{r}\right)$ of commutative group schemes over $k$ and homomorphisms $i_{r}: G_{r} \rightarrow G_{r+1}$, such that if $G_{r}=\operatorname{Spec} E_{r}, E_{r}$ is of rank $p^{r h}$ over $k$ and such that

$$
G_{r} \stackrel{i_{r}}{\longrightarrow} G_{r+1} \stackrel{p^{r}}{\longrightarrow} G_{r+1}
$$

identifies $G_{r}$ with the kernel of $p^{r}$, is called a $p$-divisible group of height $h$. 
Definition 3.5. If $G$ is a commutative divisible formal Lie group over $k$ and $G_{r}=\operatorname{kernel}\left[p^{r}\right], i_{r}: G_{r} \rightarrow G_{r+1}$ the natural inclusion, then $\left(G_{r}, i_{r}\right)$ is a $p$-divisible group $G(p)$ associated to $G$.

Tate proves in [6] that if $k$ is a perfect field of characteristic $p$, then the functor $\Gamma: G \rightarrow G(p)$ is an equivalence of the category of divisible commutative formal Lie groups over $k$ and the category of $p$-divisible local groups over $k$. Naturally, the hard part is going from the $p$-divisible group to the formal group.

Definition 3.6. Frobenius map $F$ (see [4] for more details). Let $G=\operatorname{Spec} E$ be a finite or formal commutative group scheme over a field $k$ of characteristic $p$.

Let $k^{(p)}=\left\{\lambda^{p} \mid \lambda \in k\right\}$. Let $\pi: k \rightarrow k^{(p)}$ be defined by $\lambda \mapsto \lambda^{p}$. Let $E^{(p)}=E \otimes_{\pi} k^{(p)}$ and $G^{(p)}=\operatorname{Spec} E^{(p)}$. Then there is a map $F: G \rightarrow G^{(p)}$ defined by

$$
\begin{gathered}
E^{(p)}=E \otimes_{\pi} k^{(p)} \rightarrow E \otimes_{k} k=E \\
(x \otimes \lambda) \mapsto x^{p} \otimes \lambda .
\end{gathered}
$$

$G^{(p)}$ is also a group scheme with multiplication induced from $G$ and $F$ is a group homomorphism so that kernel $F$ is well defined and is a group scheme.

We let $G^{\left(p^{r}\right)}=\left(G^{p^{r-1}}\right)^{(p)}$. If $k$ is perfect, then $E \cong E^{(p)}$ as an algebra. There is also a map $V: G^{(p)} \rightarrow G$ defined for finite group schemes by taking the $F$ map $G^{\prime} \rightarrow G^{\prime(p)}$ and dualizing $\left(G^{\prime}=\right.$ Cartier dual of $\left.G\right)$. It is well known that $F V=V F=p$ (see [4] or [2]).

Definition 3.7. Let $G$ be a formal Lie group over $k$, a field of characteristic $p$. Let ${ }_{F} G=$ kernel of $F^{r}: G \rightarrow G$. So if $G=\operatorname{Spec} k\left[\left[x_{1}, \ldots, x_{d}\right]\right]$, then

$$
{ }_{F r} G=\operatorname{Spec} B_{r} \quad \text { where } B_{r}=k\left[x_{1}, \ldots, x_{d}\right] /\left(x_{1}^{p^{r}}, \ldots, x_{d}^{p^{r}}\right) .
$$

The hyperalgebra of $G$ is inj $\lim \operatorname{Hom}_{k}\left(B_{r}, k\right)=\operatorname{Hom}_{k}\left(\operatorname{proj}_{\lim _{F}} B_{r}, k\right)$.

Proposition 3.1. Let $G$ be divisible, commutative, $G_{r}=\operatorname{Spec} A_{r}=\operatorname{kernel} p^{r}$, and $B_{r}$ as in Definition 3.7.

Then proj $\lim B_{r}=\operatorname{proj} \lim A_{r}$ so that the hyperalgebra of $G \cong \operatorname{inj} \lim \operatorname{Hom}\left(A_{r}, k\right)$ $=\operatorname{inj} \lim A_{r}^{\prime}$ where Spec $A_{r}^{\prime}=G_{r}^{\prime}$, the Cartier dual of $G$, and the map $A_{r}^{\prime} \rightarrow A_{r+1}^{\prime}$ is the map induced by $[p]: G_{r+1}^{\prime} \rightarrow G_{r}^{\prime}$.

Proof. The map [ $p$ ]: $G_{r+1} \rightarrow G_{r+1}$ induces a map $G_{r+1} \rightarrow G_{r}$, also called [ $p$ ], which is an epimorphism and which induces by duality a monomorphism

$$
G_{r}^{\prime} \stackrel{i_{r}^{\prime}}{\longrightarrow} G_{r+1}^{\prime}
$$

$\left(G_{r}^{\prime}, i_{r}^{\prime}\right)$ is a $p$-divisible group. Going from $G_{r}^{\prime}$ to $G_{r}$ we see that the map $[p]: G_{r+1}^{\prime} \rightarrow G_{r}^{\prime}$ is dual to the map $i_{r}: G_{r} \rightarrow G_{r+1}$ and we are done. See [6, §2] for more details.

Continuing with the discussion in the proof of Proposition 3.1 we see that $\left(G_{r}^{\prime}, i_{r}^{\prime}\right)$ is associated by Tate's theorem to a formal Lie group $G^{\prime}$ if $G_{r}^{\prime}$ is local. In this case $G^{\prime}=\operatorname{Spec} k\left[\left[x_{1}, \ldots, x_{d^{\prime}}\right]\right]$. 
Proposition 3.2. Let $G$ be as above so that $G_{r}^{\prime}$ is local and so $G^{\prime}$ is as above. Let ${ }_{r r} G^{\prime}=\operatorname{Spec} B_{r}^{\prime}$. Then inj $\lim _{[p]} A_{r}^{\prime}=\operatorname{inj} \lim _{F} B_{r}^{(p-r)}$ where $F: B^{\prime\left(p^{-r}\right)} \rightarrow B^{\prime p-(r+1)}$ is just

\section{Proof.}

$$
\begin{aligned}
k\left[x_{1}, \ldots, x_{d^{\prime}}\right] /\left(x_{1}^{p^{r}}, \ldots, x_{d^{\prime}}^{p^{r}}\right) & \rightarrow k\left[x_{1}, \ldots, x_{d^{\prime}}\right] /\left(x_{1}^{p^{r+1}}, \ldots, x_{d^{\prime}}^{p^{r+1}}\right) \\
x_{i} & \rightarrow x_{i}^{p} .
\end{aligned}
$$

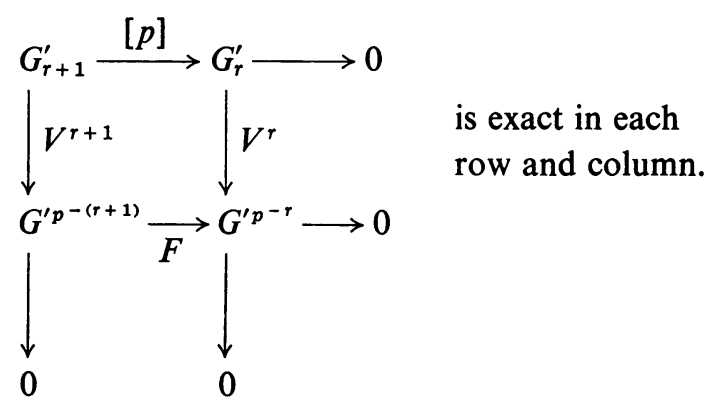

On rings,

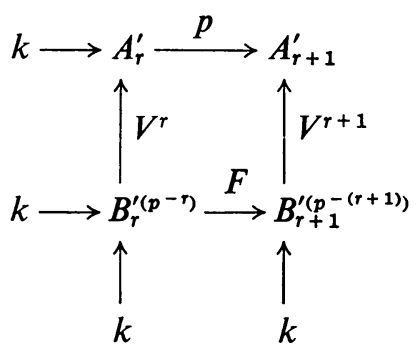

defines a map $\theta: \operatorname{inj} \lim _{F} B_{r}^{\prime(p-\eta)} \rightarrow \operatorname{inj} \lim _{p} A_{r}^{\prime}$ which is clearly injective. From now on we drop the superscript $p^{u}$ on the $A$ 's and $B$ 's.

Since $G_{r}^{\prime}$ is local for all $r, F^{s} A_{r}^{\prime}$ in $A_{r+s}^{\prime}$ is the ideal $\left(x_{1}^{p^{s}}, \ldots, x_{d^{\prime}}^{p s}\right)$ in

$$
A_{r+s}^{\prime}=k\left[x_{1}, \ldots, x_{d^{\prime}}\right] /\left(x_{1}^{p^{r+s}}, \ldots, x_{d^{\prime}}^{p^{r+s}}\right) \text {. }
$$

Since $\left(G_{r}^{\prime}, i_{r}^{\prime}\right)$ is $p$-divisible, $\operatorname{dim}_{k}\left(A_{r+s}^{\prime} / V^{r} B_{r+s}^{\prime}\right)<c(r)$ for some constant $c(r)$ independent of $s$.

Thus for $s$ large enough $F^{s} A_{r}^{\prime} \subseteq V^{r} B_{r+s}^{\prime}$ in $A_{r+s}^{\prime}$ which implies $V^{s} F^{s} A_{r}^{\prime} \subseteq V^{s} V^{r} B_{r+s}^{\prime}$ so $p^{s} A_{r}^{\prime} \subseteq V^{r+s} B_{r+s}^{\prime}$ in $A_{r+s}^{\prime}$ for $s$ large enough. So $\theta$ is surjective and hence is an isomorphism.

COROLlary. If $G$ is a commutative divisible formal Lie group over a perfect field $k$ with local dual (meaning $G_{r}^{\prime}$ is local) then the hyperalgebra of $G$ is isomorphic to $k\left[Q_{p} / Z_{p}^{\oplus d^{\prime}}\right]$ as an algebra, for some integer $d^{\prime}$.

Proposition 3.3. Let $B_{r}^{\prime}=k\left[x_{1}, \ldots, x_{d^{\prime}}\right] /\left(x_{1}^{p^{r}}, \ldots, x_{d^{\prime}}^{p^{r}}\right)$ as in Proposition 3.2 and let

$$
\begin{aligned}
F: B_{r}^{\prime} & \rightarrow B_{r+1}^{\prime} \\
x_{i} & \rightarrow x_{i}^{p}
\end{aligned}
$$


be as before. Then $F$, which is a homomorphism of Hopf algebras, induces a graded ring homomorphism $F^{*}$.

$$
\begin{aligned}
k\left[U_{1}^{(r+1)}, \ldots, U_{d^{\prime}}^{(r+1)}, \ldots, V_{1}^{(r+1)}, \ldots, V_{d^{\prime}}^{(r+1)}\right] & =H^{\cdot}\left(B_{r+1}, k\right) \stackrel{F^{*}}{\longrightarrow} H^{\cdot}\left(B_{r}, k\right) \\
& =k\left[U_{1}^{(r)}, \ldots, U_{d^{\prime}}^{(r)}, V_{1}^{(r)}, \ldots, V_{d^{\prime}}^{(r)}\right]
\end{aligned}
$$

$V_{i}^{(r)}$ of degree $1, V_{i}^{(r)^{2}}=0, U_{i}^{(r)}$ of degree 2, as in the Corollary to Proposition 2.4, and $F^{*} U_{i}^{(r+1)}=U_{i}^{(r)}, F^{*} V_{i}^{(r+1)}=0$.

Proof. The proof is another calculation involving resolutions. Let $X_{\text {. }}^{(r)}=$ $B_{r}^{\prime} \otimes_{k} \Lambda\left[T_{1}^{(r)}, \ldots, T_{d^{\prime}}^{(r)}\right] \otimes_{k} \Gamma\left[S_{1}^{(r)}, \ldots, S_{d^{\prime}}^{(r)}\right]$ as in $\S 2$.

The map $h^{(r)}: B_{r}^{\prime} \rightarrow B_{r+1}^{\prime}$ of Hopf algebras induce a map

of resolutions and

$$
X_{\cdot}^{(r)} \stackrel{h^{(r)}}{\longrightarrow} X_{\cdot}^{(r+1)}
$$

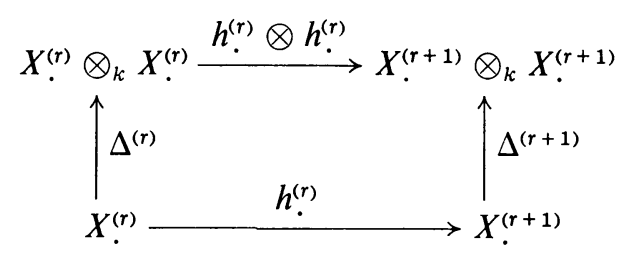

commutes up to homotopy since it commutes in dimension 0 . We only need to compute $h^{(r)}$ on the generators of $X^{(r)}$

$$
\begin{aligned}
h_{1}^{(r)}\left(T_{j}^{(r)}\right) & =s h_{0}^{(r)} \partial T_{j}^{(r)}=s h_{0}^{(r)}\left(x_{j}\right)=s\left(x_{j}^{p}\right)=x_{j}^{p-1} T_{j}^{(r+1)}, \\
h_{2}^{(r)}\left(S_{j}^{(r)}\right) & =s h_{1}^{(r)} \partial S_{j}^{(r)}=s h_{1}^{(r)} x_{j}^{p^{r}-1} T_{j}^{(r)}=s h_{0}\left(x_{j}^{p^{r}-1}\right) h_{1}\left(T_{j}^{(r)}\right)=s\left(x_{j}^{p^{r+1}-p} \cdot x_{j}^{p-1} T_{j}^{(r+1)}\right) \\
& =s\left(x_{j}^{p^{r+1}-1} T_{j}^{(r+1)}\right)=S_{j}^{(r+1)}, j=1, \ldots, d^{\prime} .
\end{aligned}
$$

To finish the proof we dualize, remembering that $V_{j}^{(r)}$ is dual to $T_{j}^{(r)}, U_{j}^{(r)}$ is dual to $S_{j}^{(r)}$.

THEOREM 3.1. If $G$ is a p-divisible commutative formal Lie group over a perfect field with local dual and $\mathcal{O}$ is the functor $\mathscr{C} \leadsto \operatorname{Rings,} \mathcal{O}(\operatorname{Spec} A)=A$. Then

is an isomorphism.

$$
\operatorname{Sym}\left(H_{\text {sym }}^{2}(G, \mathcal{O})\right) \rightarrow H^{\cdot}(G, \mathcal{O})
$$

Proof. Let $G=\operatorname{Spf} E$, then $C^{n}(G, \mathcal{O})=\mathcal{O}\left(G^{x n}\right)=E^{\hat{\otimes} n}$. The ^ refers to completion of the tensor product with respect to the $m$-adic topology.

$$
E^{\otimes} n=\operatorname{proj} \lim E_{r}^{\otimes n}=\operatorname{proj} \lim \operatorname{Hom}_{k}\left(A_{r}^{\otimes n}, k\right)=\operatorname{Hom}_{k}\left(\operatorname{inj} \lim _{p} A_{r}^{\otimes n}, k\right)
$$

where Spec $E_{r}=$ kernel $p^{r}$ as usual and $A_{r}$ is the dual Hopf algebra to $E_{r}$. If Spec $B_{r}$ $={ }_{r r} G^{\prime}, G^{\prime}$ being the dual group to $G$, then $E^{\hat{\otimes} n}=$ Hom (inj $\lim _{F} B_{r}^{\otimes n}, k$ ) by Proposition 3.2. So the problem reduces to showing

$$
\text { proj } \lim _{F^{*}} H^{\cdot}\left(B_{r}, k\right)=H^{\cdot}\left(\operatorname{inj} \lim B_{r}, k\right) ;
$$


for once we show this, Proposition 3.3 will give the desired result. But the MittagLeffler condition of Dieudonné-Grothendieck [7, III,0.8] will be sufficient for $(*)$ if it is not otherwise clear.

\section{BIBLIOGRAPHY}

1. H. Cartan and S. Eilenberg, Homological algebra, Princeton Univ. Press, Princeton, N. J., 1956.

2. M. Demazure and A. Grothendieck, Schémas en groups. Fasc. 4: Exposés 12 à 14. Séminaire de géométrie algébrique, 1963-1964, Inst. Hautes Études Sci., Paris, 1964.

3. G. Efroymson, Cohomology rings of certain formal groups, Thesis, Harvard, 1967.

4. Yu. Manin, Theory of commutative formal groups over fields of finite characteristic, Uspehi Mat. Nauk 18 (1963), no. 6 (114), 3-90= Russian Math. Surveys 18 (1963), no. 6, 1-83.

5. F. Strooker Oort, The category of finite bialgebras over a field, Indag. Math. 29 (1967), 163-169.

6. J. Tate, $p$ divisible groups in proceedings of a conference on local fields, Springer-Verlag, New York, 1967, pp. 158-183.

7. A. Grothendieck, Éléments de géométrie algébrique. III. Étude cohomologique des faisceaux cohérents. I, Inst. Hautes Études Sci. Publ. Math. No. 11, 1961.

INDIANA UNIVERSITY,

BLOOMINGTON, INDIANA

The UNiversity of New MeXico, Albuquerque, New Mexico 